\title{
The Role of the Affective and Emotional Composite Temperament Model in Predicting Negative Attitudes towards Delinquency in Students
}

\author{
Parisa Moradi Kelardeh*, Sajjad Basharpour
}

Department of Psychology, University of Mohaghegh Ardabili, Ardabil, Iran

\section{A BSTRACT}

Introduction: This study aimed to investigate the predictive role of the affective and emotional composite temperament (AFECT) model concerning negative attitudes toward delinquency in secondary high school students. Materials and Methods: This study was a descriptive- correlational investigation and its statistical population was all secondary high school students of Ardabil city. 221 students were selected using the multi- stage cluster sampling method. Data was collected by Affective and Emotional Temperament questionnaires, Lara (2012) and Questionnaire of Delinquency, Fazli (2010). Pearson correlation and stepwise regression were used to analyze data. Results: The results revealed that the anger, inhibition, and sensitivity of the AFECT model have a negative and significant relationship with a negative attitude towards delinquency, and control. Furthermore, coping has a significant positive relationship with negative attitudes towards delinquency. Among the affective temperaments, anxiety, obsession, euthymia, and hyperthermia have a positive and significant relationship with a negative attitude towards delinquency. Moreover, apathetic, cyclothymic, volatile, and euphoric had a negative and significant relationship with negative attitudes towards delinquency. Conclusion: Our data have important implications for use of the AFECT model with the aim of identifying groups at risk of delinquency and can be used to design preventive interventions to reduce the occurrence of delinquent behavior in students.

*Corresponding Author: Parisa Moradi Kelardeh

Email: pari.moradi71@gmail.com 


\section{نقش مدل تركيبى سرشتهاى عاطفى و هيجانى در ييشبينى نكَرشهاى منفى به بزهكارى در دانش آموزان}

"بر يسا مرادى كلارده"، سجاد بشر يور

كروه روانشناسى، دانشاءه محقق اردبيلى، اردبيل، ايران

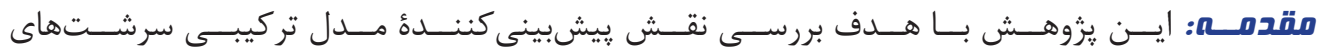

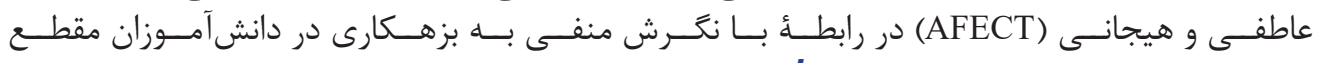

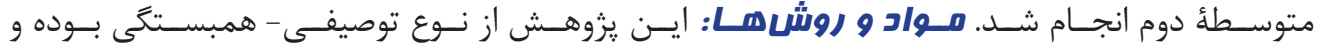

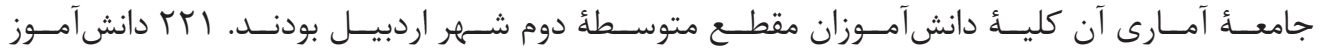

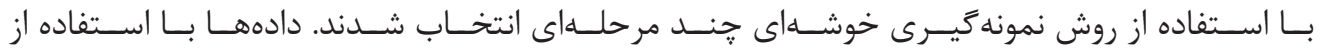

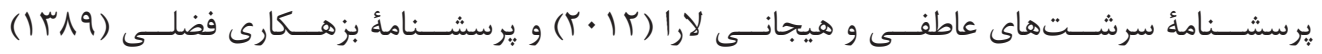

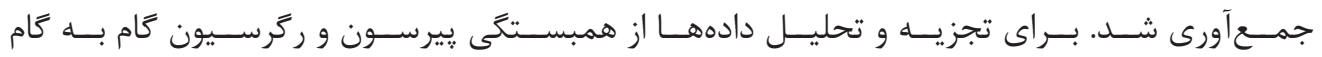

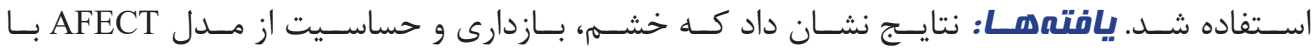

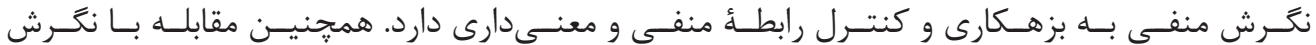

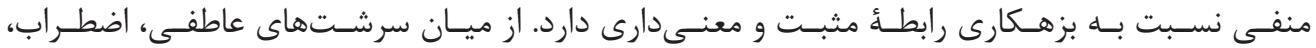

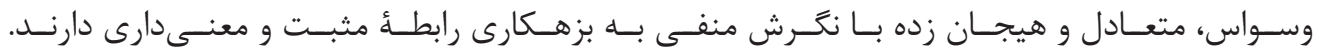

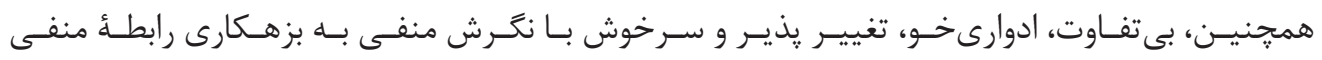

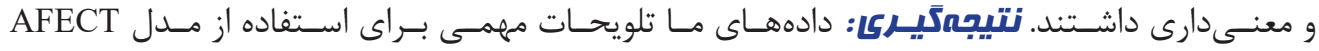

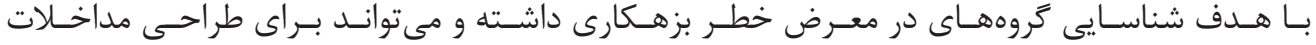

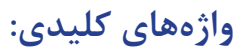

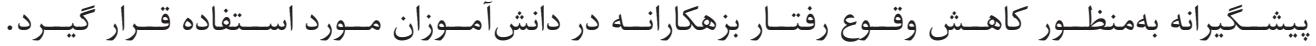




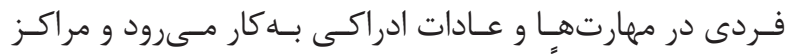

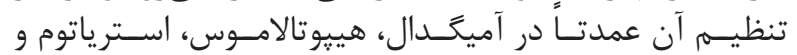

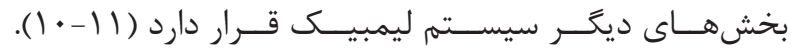

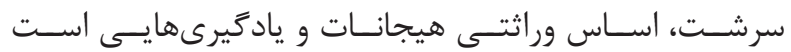

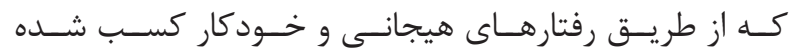

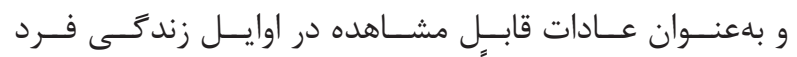

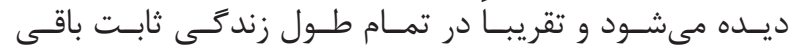

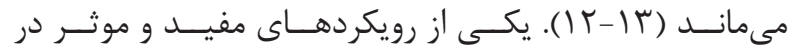

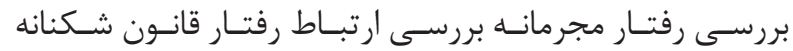

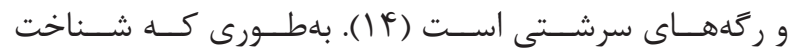

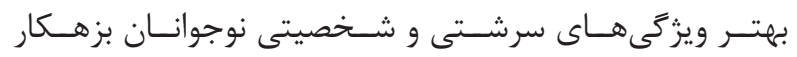

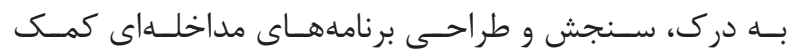

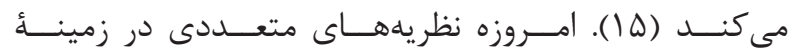

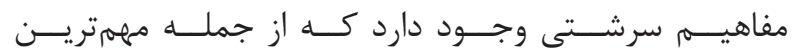

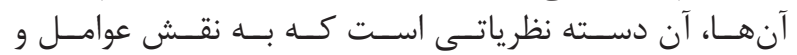

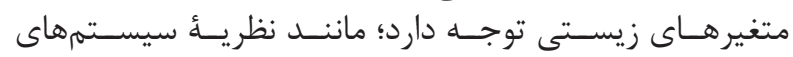

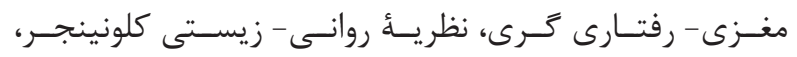

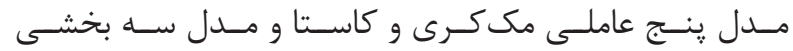

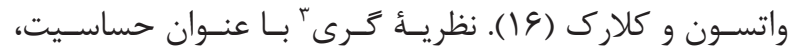

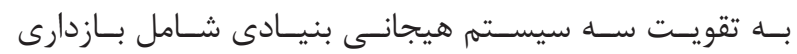

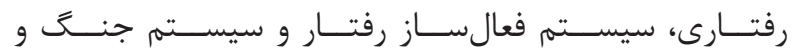

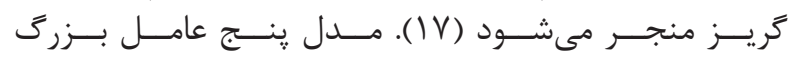

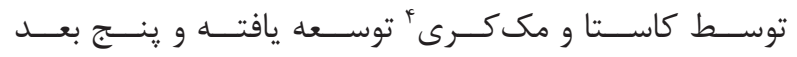

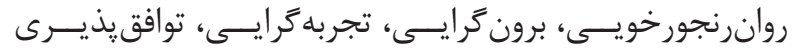

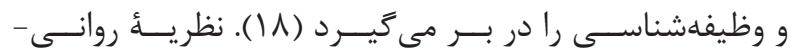

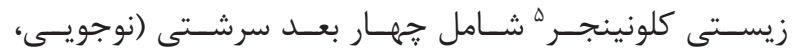

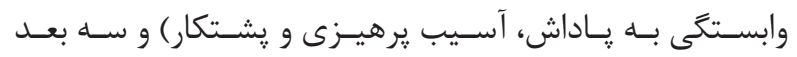

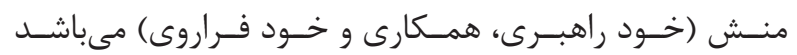

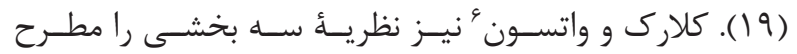

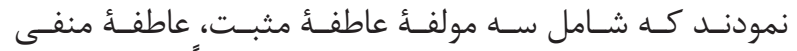

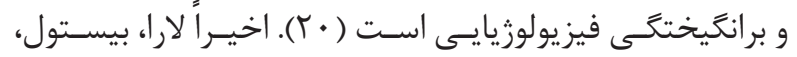

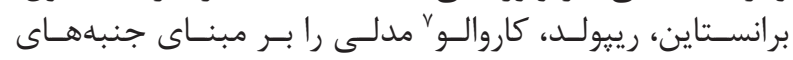

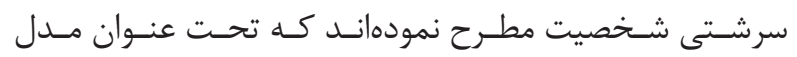

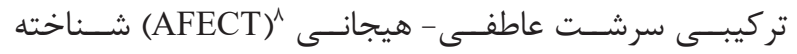

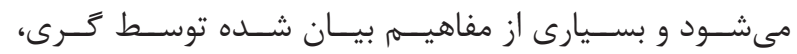

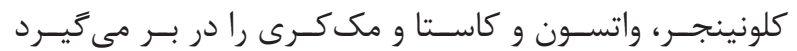

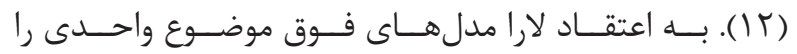

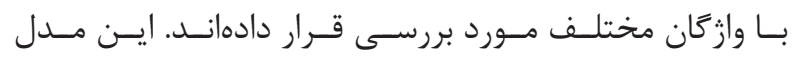

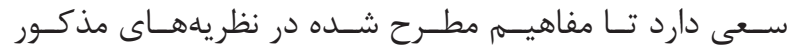

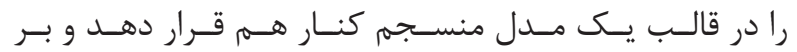

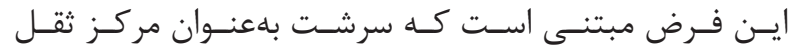

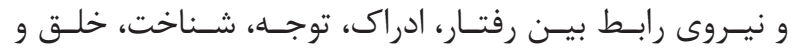

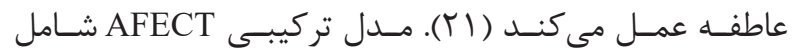

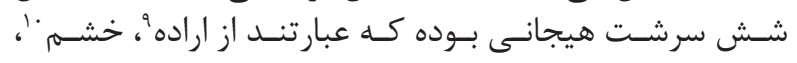

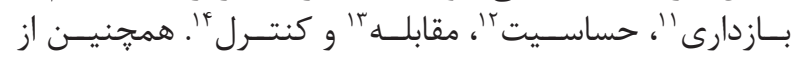

${ }^{1}$ Delinquency

${ }^{2}$ Temperament

${ }^{3}$ Gray

${ }^{4}$ Costa \& Mc Crae

${ }^{5}$ Cloninger

${ }^{6}$ Clark \& Watson

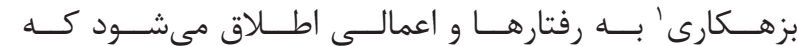

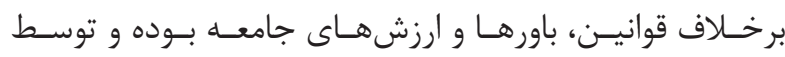

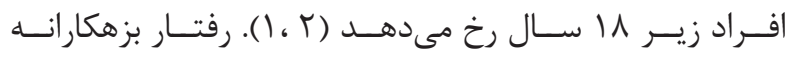

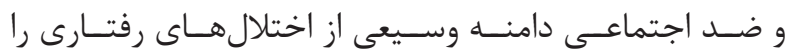

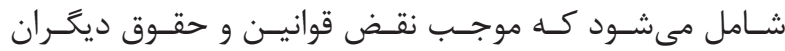

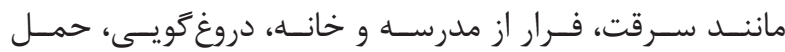

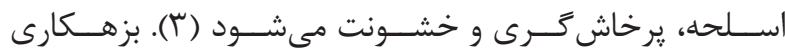

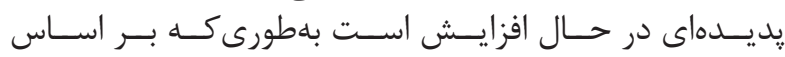

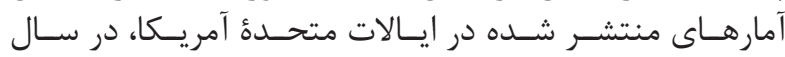

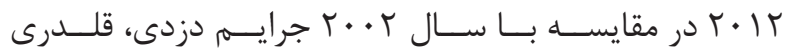

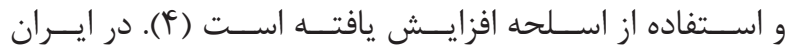

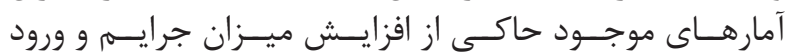

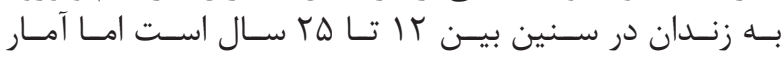

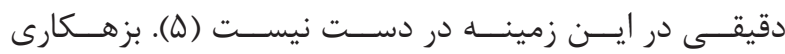

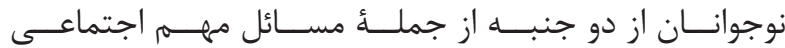

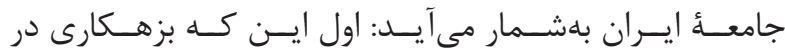

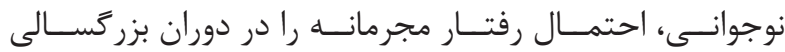

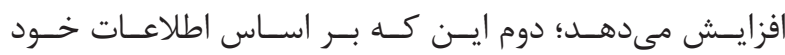

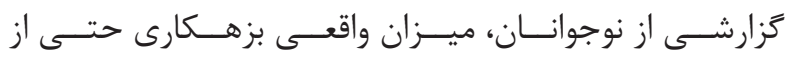

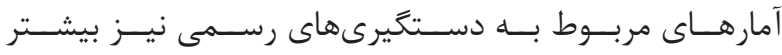

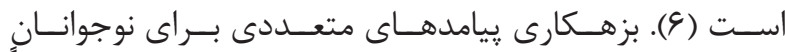

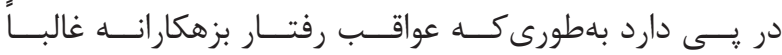

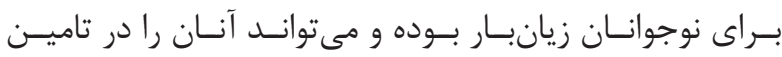

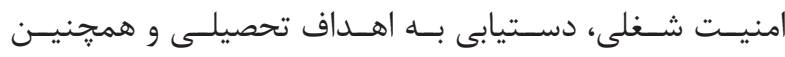

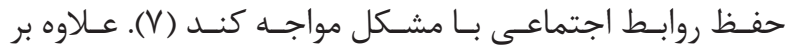

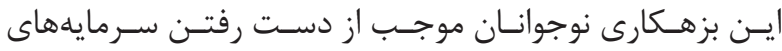

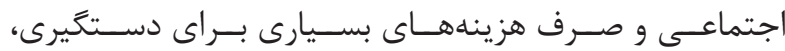

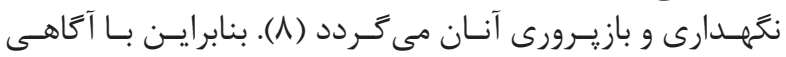

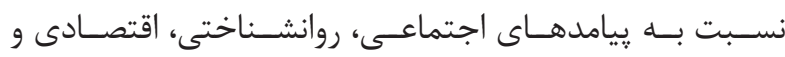

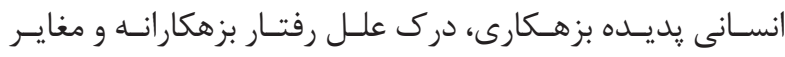

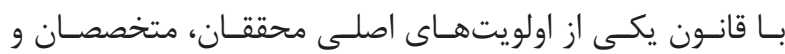

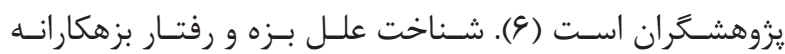

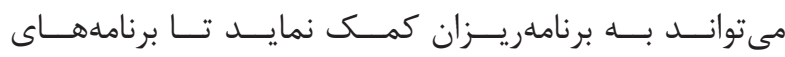

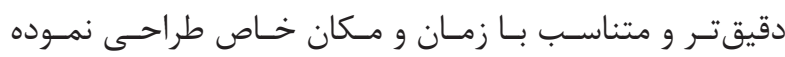

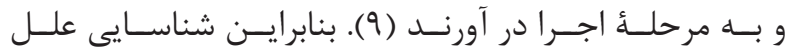

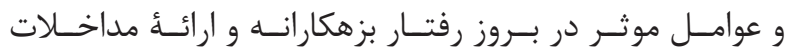

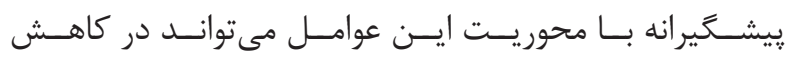

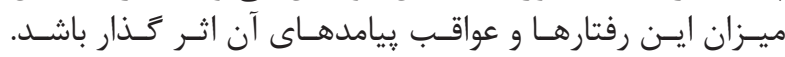

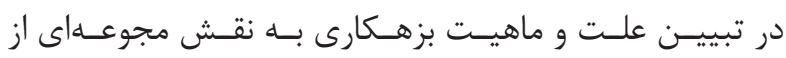

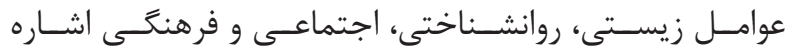

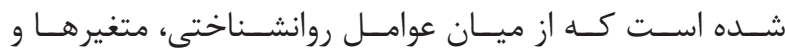

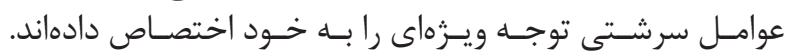

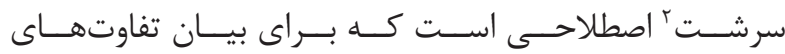

7. Lara, Bisol, Brunstein, Reppold, DeCarvalho, and Ottoni

${ }^{8}$ Affective and Emotional Composite Temperament Model

${ }^{9}$ Volition

${ }^{10}$ Anger

${ }^{11}$ Inhibition

${ }^{12}$ Sensivity

${ }^{13}$ Coping

${ }^{14}$ Control 


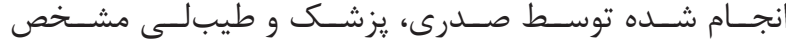

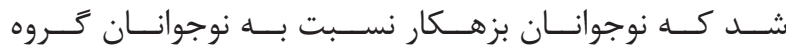

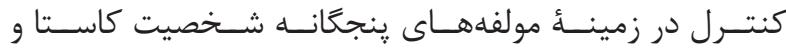

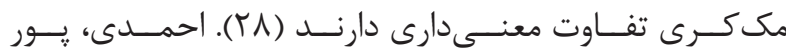

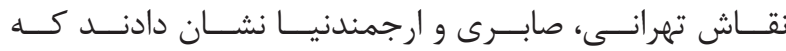

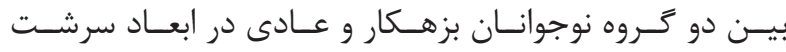

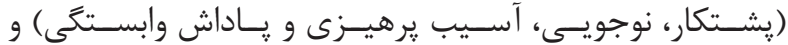

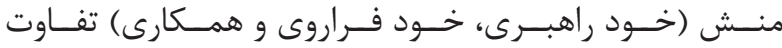

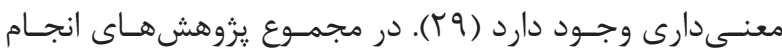

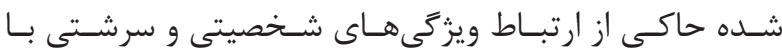

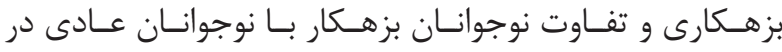

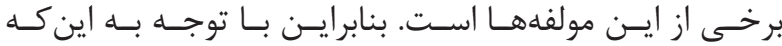

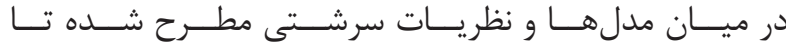

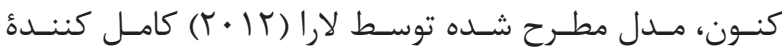

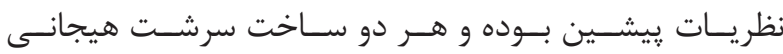

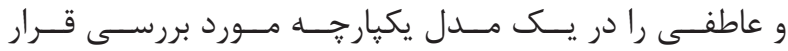

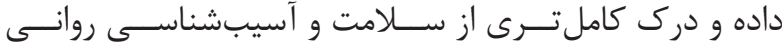

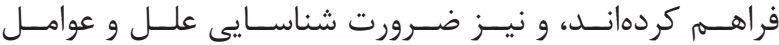

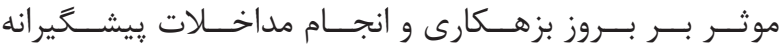

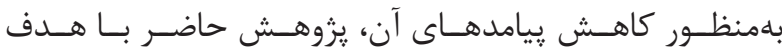

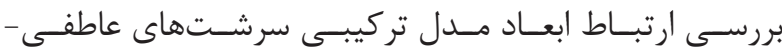

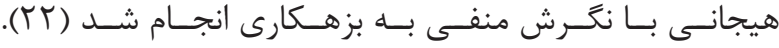

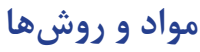

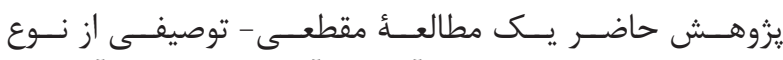

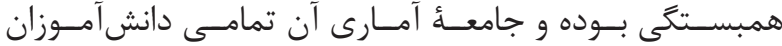

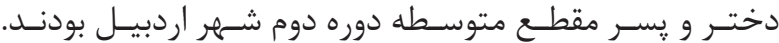

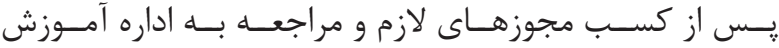

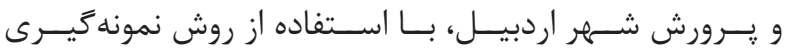

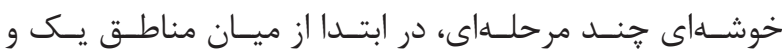

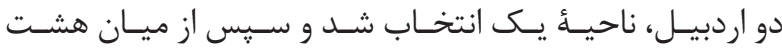

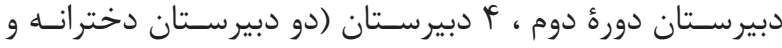

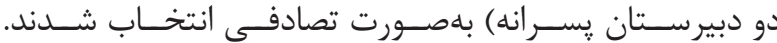

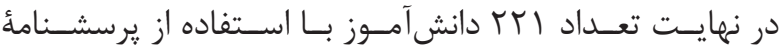

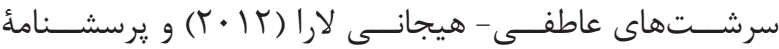

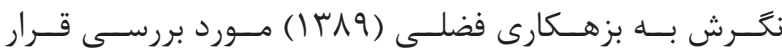

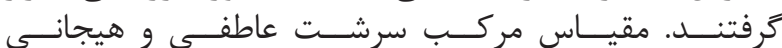
هـ (AFECT)

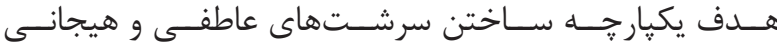

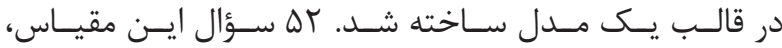

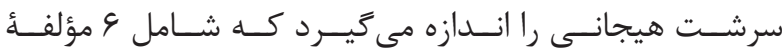

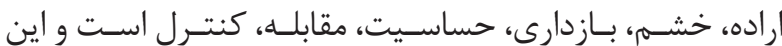

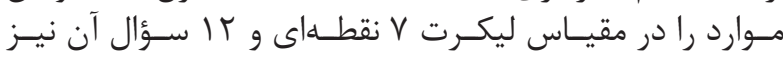

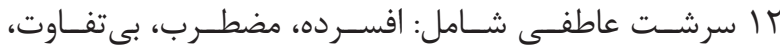

\footnotetext{
${ }^{15}$ Depressive

${ }^{16}$ Anxious

${ }^{17}$ Apathetic

${ }^{18}$ Obsessive

${ }^{19}$ Hyperthimia

${ }^{20}$ Euthymia

${ }^{21}$ Stable

${ }^{22}$ Volatile
}

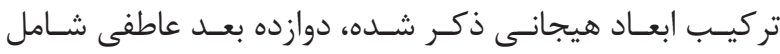

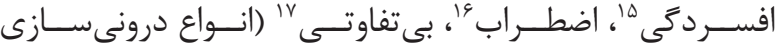

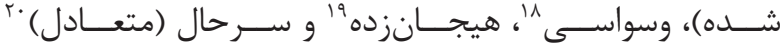

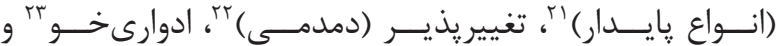

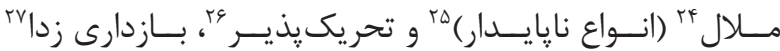

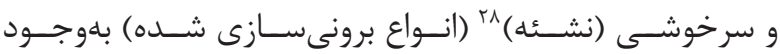

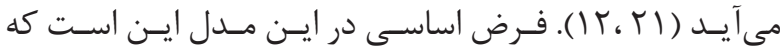

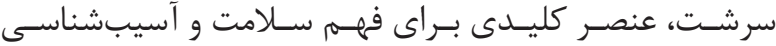

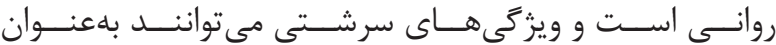

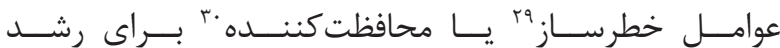

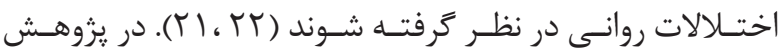

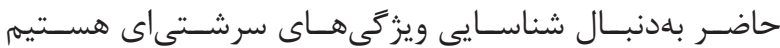

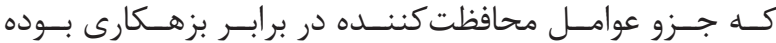

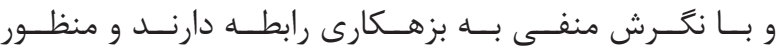

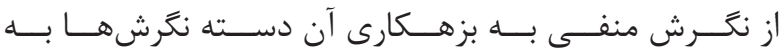

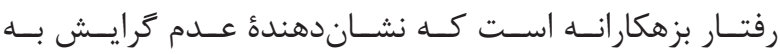

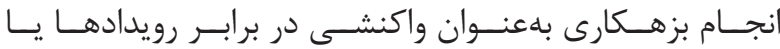

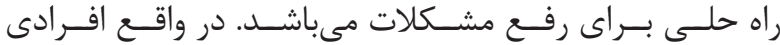

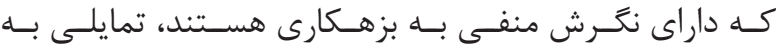

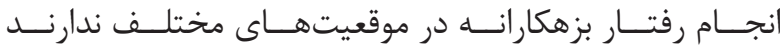

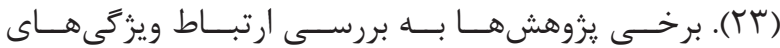

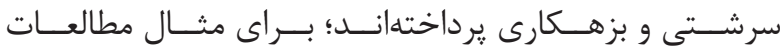

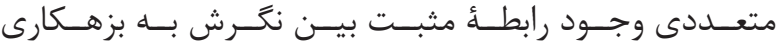

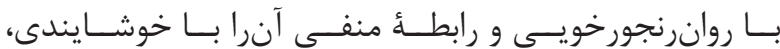

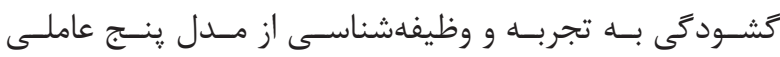

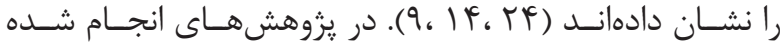

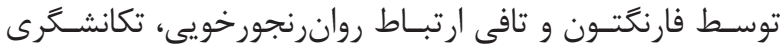

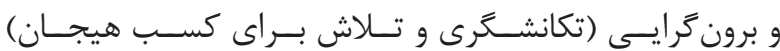

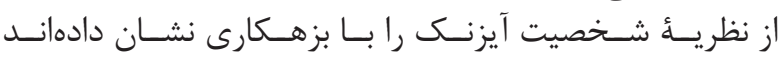

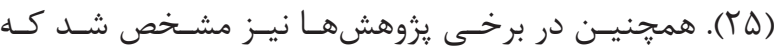

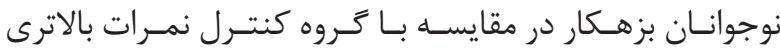

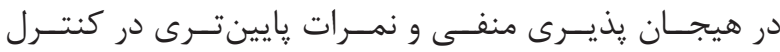

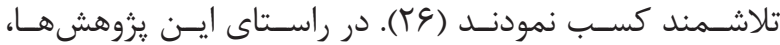

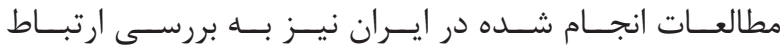

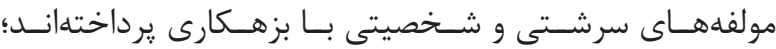

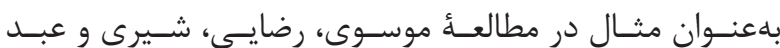

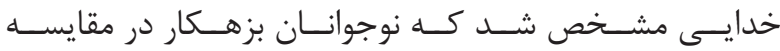

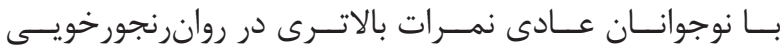

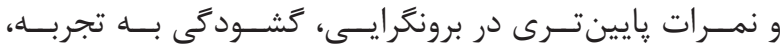

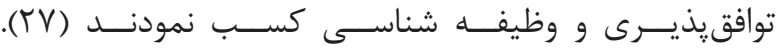

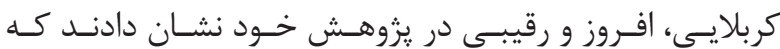

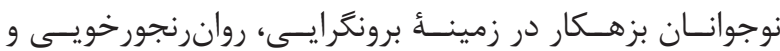

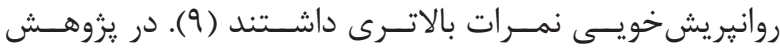

\footnotetext{
${ }^{23}$ Cyclothymic

${ }^{24}$ Dysphric

${ }^{25}$ Unstable

${ }^{26}$ Irritable

${ }^{27}$ Disinhibited

${ }^{28}$ Euphoric

${ }^{29}$ Risk factor

${ }^{30}$ Protective factor
} 


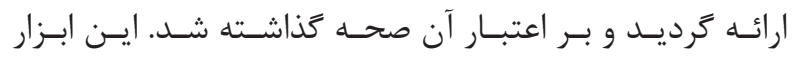

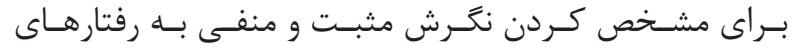

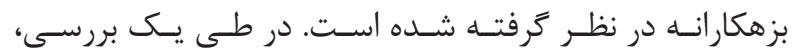

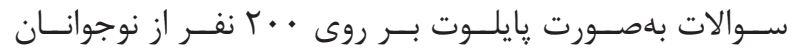

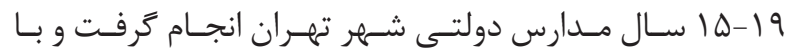

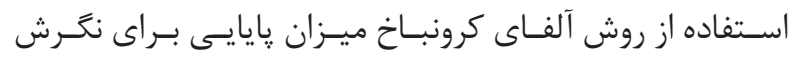

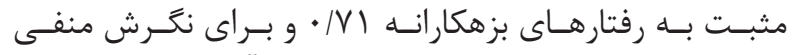

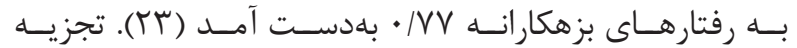

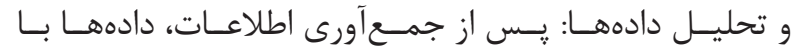

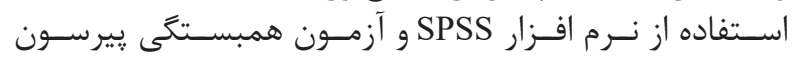

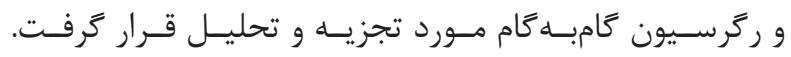

يافتهها

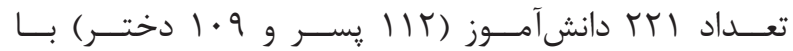

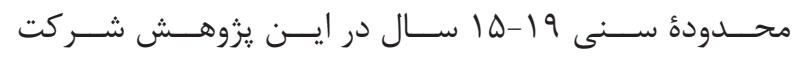

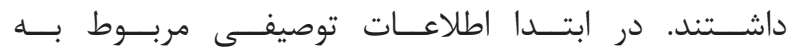

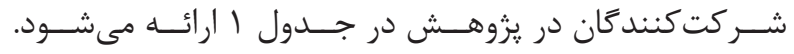

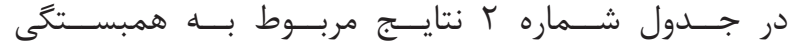

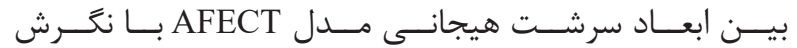

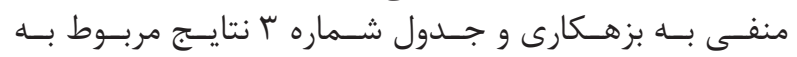

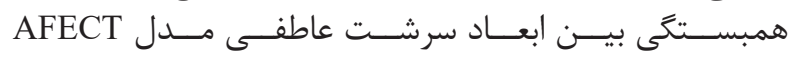

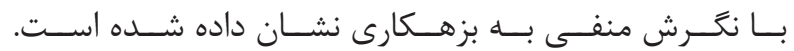

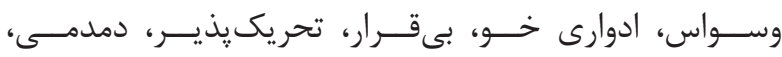

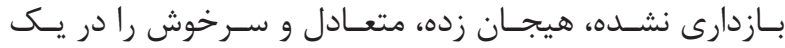

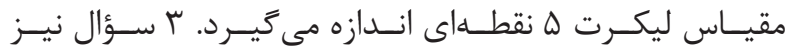

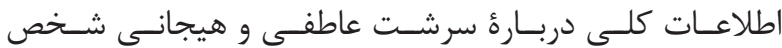

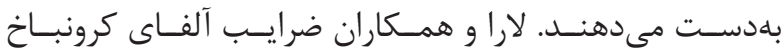

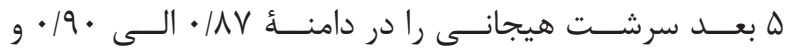

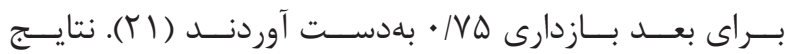

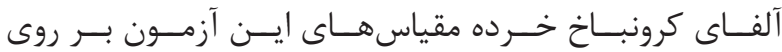

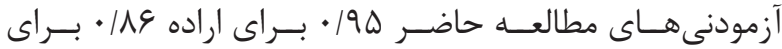

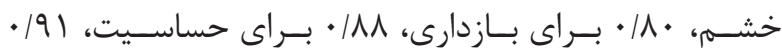

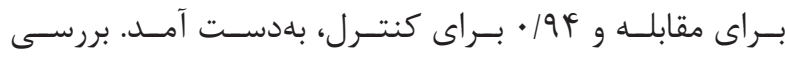

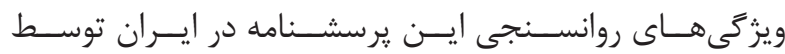

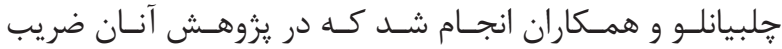

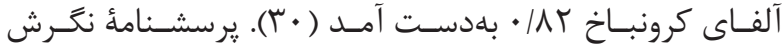

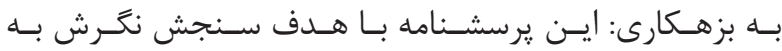

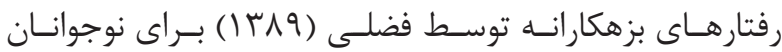

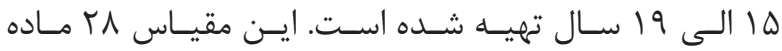

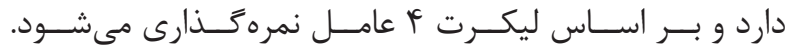

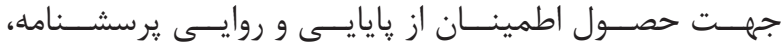

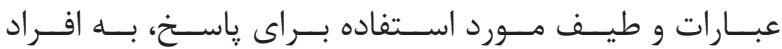
صاحسب نظــر در رابطــه بــا موضـوع مـورد مطالعـهـ (اسـتادان

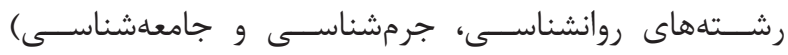

جدول ا- اطلاعات توصيفى مربوط به شركت كنندكان در يثوهش

\begin{tabular}{|c|c|c|c|}
\hline ميانَين & فراوانى & شاخص & متغير \\
\hline & 114 & مرد & \multirow{2}{*}{ جنسيت } \\
\hline & 1.9 & زن & \\
\hline $19 / r \Delta$ & 110 & Vا-هال سال & \multirow{2}{*}{ سن } \\
\hline $1 N / 1 T$ & 1.9 & 9 IV-1 سال & \\
\hline & NT & اول متوسطه & \multirow{3}{*}{ هايةُ تحصيلى } \\
\hline & $\vee \Delta$ & دوم متوسطه & \\
\hline & gf & سوم متوسطه & \\
\hline & THI & & تعداد كل \\
\hline
\end{tabular}

جدول r- ماتريس همبستخى ابعاد سرشت هيجانى مدل AFECT با نكرش منفى به بزهكارى

\begin{tabular}{|c|c|c|c|c|c|c|c|}
\hline كنترل & مقابله & حساسيت & 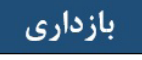 & خشم & 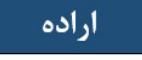 & نَّرش منفى به بزهكارى & متغير \\
\hline & & & & & & 1 & نكَر منفى به بزهكارى \\
\hline & & & & & 1 &.$/ \cdot \Delta T$ & اراده \\
\hline & & & & 1 & $-\cdot|r| \notin * *$ & $-\cdot / 194 * *$ & خشم \\
\hline & & & 1 & $\cdot 1 \cdot \mathrm{rF}$ & -.1 .99 & $-\cdot / 119 * *$ & بازدارى \\
\hline & & 1 & •/Кт人** &.$/ 19 V^{* *}$ & $-\cdot / 1 \cdot r * *$ & $-\cdot / 1 \cdot r * *$ & حساسيت \\
\hline & 1 & $\cdot|\cdot r|$ & $-\cdot / 1 \cdot 1 * *$ & $\cdot|\cdot r|$ & $\cdot / \cdot r$ & . $\mid$ ४४q** & مقابله \\
\hline 1 &.$/ 911^{* *}$ & $\cdot 1 \cdot 11$ & $-\cdot / \cdot 91$ & $\cdot / \cdot \wedge \mathrm{V}$ &.$- / \cdot 1 r$ & $\cdot \mid \Delta \& \wedge * *$ & كنترل \\
\hline
\end{tabular}


جدول بـ- ماتريس همبستخى ابعاد سرشت عاطفى مدل AFECT با نكَرش منفى به بزهكارى

\begin{tabular}{|c|c|c|c|c|c|c|c|c|c|c|c|c|c|}
\hline سرخوشى & بازازى زدا & تحريكيذير & هيجانزه & سر حال & وسواسى & تفيريذير & ملال & ادوارىخو & بيقتفاوت & مضطرب & افسر ده & نكَرش منفى به بزهكارى & متغير \\
\hline & & & & & & & & & & & & 1 & نكُرش منفى به بزهكارى | \\
\hline & & & & & & & & & & & 1 & $\% r$ & 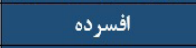 \\
\hline & & & & & & & & & & 1 & $\cdot|\Psi Y|^{* *}$ & $.|| r \mid * *$ & مضطرب \\
\hline & & & & & & & & & 1 & . /raq"* & $. / 1 / 9^{* *}$ &.$- / 1$ frets & بعتفاوت \\
\hline & & & & & & & & 1 & . Kry &.$/ \mu q v^{* *+}$ & $\cdot / 1 \Delta \Delta^{* * *}$ &.$- / 111 \%$ & ادوارىخو \\
\hline & & & & & & & 1 & $\cdot / 1 \cdot 1^{* *}$ & . ITY &.$/ \Gamma M \Lambda^{2=}$ & $\cdot / r \Lambda \cdot=*$ &.$- / . r T$ & ملال \\
\hline & & & & & & 1 &. $\mid f \xi q=$ & 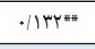 & . & $. / 19 \mathrm{~V}=*$ &. $\mid \mu q \varepsilon=\#$ &.$- / 110^{* * *}$ & تغييريذير \\
\hline & & & & & 1 & .1 .99 & . $/ / r^{2+*}$ & $-.1 . \mu f$ &.$/ \Delta \cdot$ & $\cdot \mid r \cdot 9=4$ & 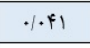 & $. / 11 \%=$ & وسواسى \\
\hline & & & & 1 & - IrrTus & $-*|+r|$ & +1.49 &.$- / . r \mu$ & $-\cdot / 1 \cdot \Delta$ & .1 .94 & $-* 1.49$ & 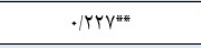 & متعادل \\
\hline & & & 1 & $. / T Y Y=*$ &.$|r \cdot|^{n+*}$ & $-\cdot / / Y f= \pm$ & $.1 . \Delta 9$ & $-\% . \%$ & $-\cdot / 1 \Delta 9$ &.$- / .9$ & $--/|f|=*$ & . & هيجانزده \\
\hline & & 1 & 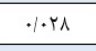 & $\% \cdot \Delta$ & - /194*t & $\cdot|r| \Lambda^{* * *}$ & $.|\mu| r *=$ & ./IMT"* & . Krs & ./IYY=" & $\cdot / r 19^{* *}$ & $\cdot / \cdot \wedge f$ & تحريكيذير \\
\hline & 1 & $. / r y 9=$ &.$- / 119$ at & $-* 1 \cdot 19$ & .1 .90 & $\cdot r \cdot v^{* * *}$ & . $/ \mathrm{rM}=$ & $\cdot / \| \Delta v^{E *}$ &.$|49|$ & $. / 10 . * *$ & $\cdot / 1 \Delta v^{* *}$ &.$- / . \Delta V$ & بازدارىزدا \\
\hline 1 &.$/ \% Y V^{* 4 *}$ & . MYrum &.$- / . \Delta \mu$ & $-\cdot / / \mid \uparrow \&+4$ & $-* \% \vee$ & - $/$ rrg*\# & . $/ 1 / q^{* *}$ & $\cdot / 11 \Lambda^{* * *}$ &.$/ 1 \% \Delta$ & $+|+r|$ & $+\cdots 4$ &.$- / 1 \Delta r^{t+z}$ & سرخوشى \\
\hline
\end{tabular}

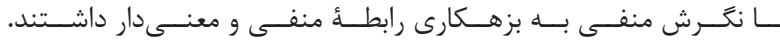

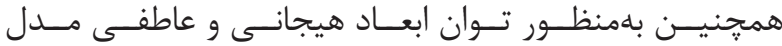
AFECT

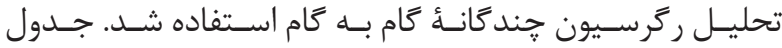

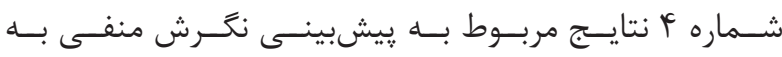

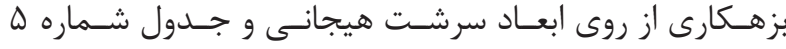

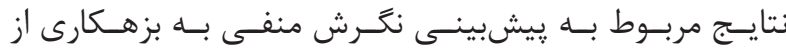

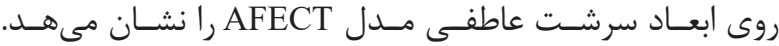

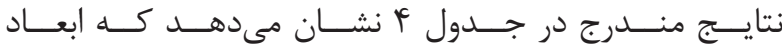

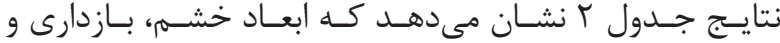

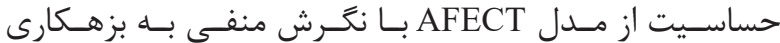

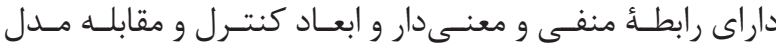

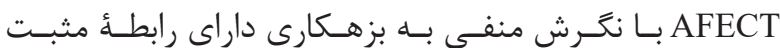

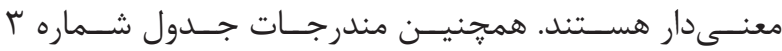

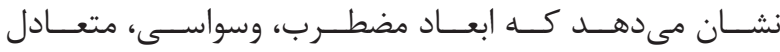

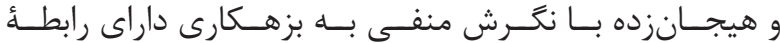

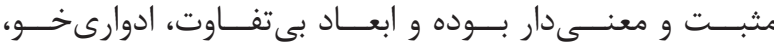

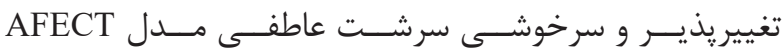

جدول F- تحليل ركرسيون ابعاد سرشت هيجانى مدل AFECT در ارتباط با نكَرش منفى به بزهكارى

\begin{tabular}{|c|c|c|c|c|c|}
\hline $\mathbf{R}_{\mathbf{2}}$ & $\mathbf{R}$ & سطح معنى دارى & $\mathbf{t}$ & $\boldsymbol{\beta}$ & متغير \\
\hline & & טגוא. & .1994 & $\cdot / \cdot \Delta \cdot$ & اراده \\
\hline & & $\cdot 1 \cdots$ & $-r|\Delta f|$ & $-\cdot / 1 \vee q$ & خشم \\
\hline$\cdot / f \cdot \varphi$ & .1949 & $\cdot / r \wedge \mathrm{V}$ & $-1 / .9 \Lambda$ & $-\cdot 1 \cdot \Delta \varphi$ & بازدارى \\
\hline & & $.1 \cdot \mathrm{rd}$ & - T/TEY &.$- / 11 \mathrm{~V}$ & حساسيت \\
\hline 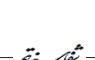 & & $\cdot 1 \cdots$ & $\mid r / 9 \Delta \Lambda$ & . ISFT & مقابله \\
\hline
\end{tabular}

جدول هـ- تحليل ركرسيون ابعاد سرشت عاطفى مدل AFECT در ارتباط با نكَرش منفى به بزهكارى

\begin{tabular}{|c|c|c|c|c|c|}
\hline $\mathbf{R}_{\mathbf{2}}$ & $\mathbf{R}$ & سطح معنى دارى & $\mathbf{t}$ & ; & متغير \\
\hline & & $\cdot / 21 \cdot$ & $\cdot / r \mid \cdot$ & $\cdot 1 \cdot$ If & افسرحه \\
\hline & & $1 / \Delta T$. & I/QT. & $\cdot / 1 \cdot 1$ & مضطرب \\
\hline & & $-1 / 1+4$ & $-1 / 1 T^{\prime}$ & $-\cdot M r \cdot$ & بى تفاوت \\
\hline & & $-1 / 9 \cdot \Delta$ & $-1 / 9 \cdot \Delta$ & $-\cdot / 1 \cdot \Delta$ & ادوارى خو \\
\hline & & $-\cdot /$ TFI & - ITFI &.$- / \cdot t r$ & ملال \\
\hline$\cdot / \cdot \Delta 1$ &.$/ t t 2$ & $-1 / 8 \Delta \lambda$ & $-1 / 9 \Delta \wedge$ & $-\cdot / 1 \cdot$ & تغيير هذير \\
\hline & & ./9rद & ./9rद & . & وسواسى \\
\hline & & H/FFt & thet &.$/ t t 2$ & متعادل \\
\hline & & $1 / \cdot r d$ & $1 / 42$ & $\cdot 1 \cdot V r$ & هيجانزده \\
\hline & & $1 / K \Delta V$ & I/TUV & $\cdot / \cdot N T$ & تحريكس يذير \\
\hline & & $-/ \Delta S T$ & -/DST & $-\cdot 1 \cdot 42$ & بازدارى زدا \\
\hline & & $-1 / \wedge F \wedge$ & -1/人F人 &.$- / 1 T 4$ & سرخوشى \\
\hline
\end{tabular}




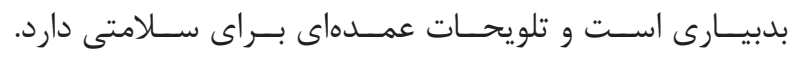

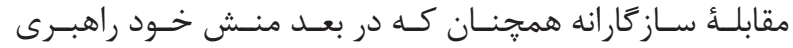

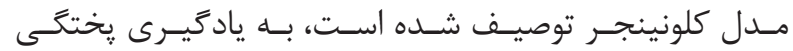

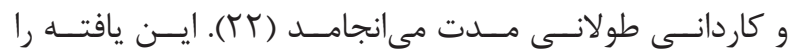

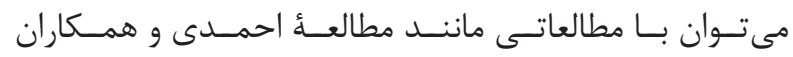

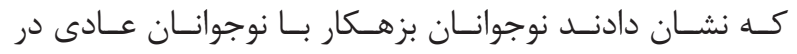

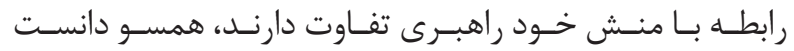

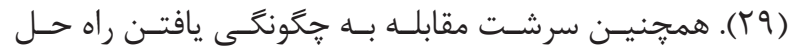

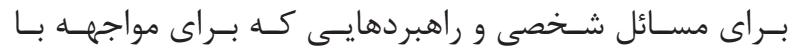

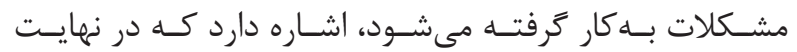

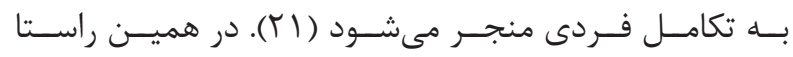

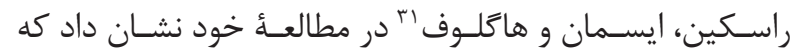

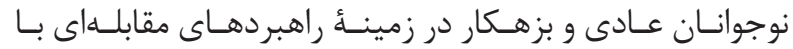

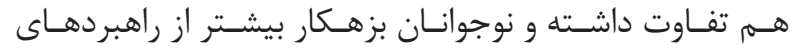

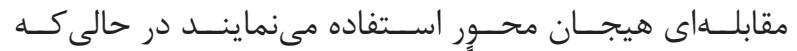

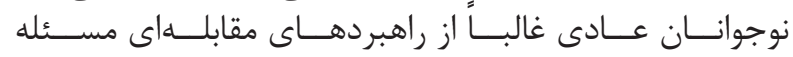

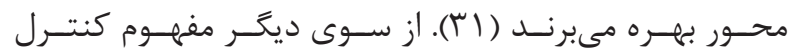

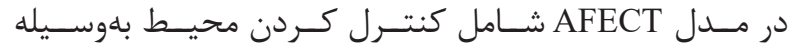

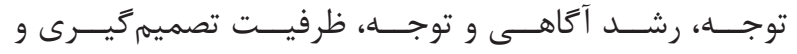

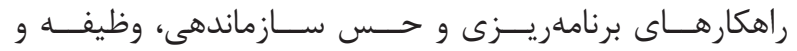

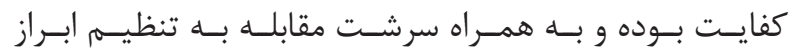

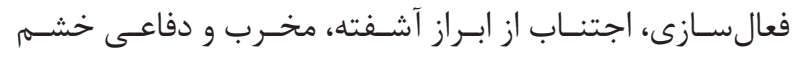

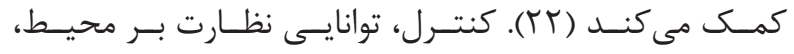

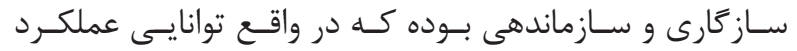

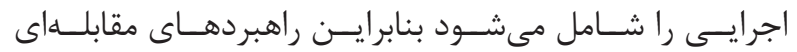

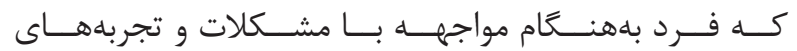

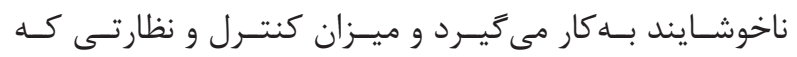

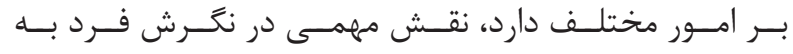

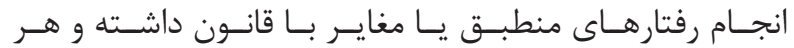

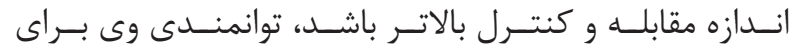

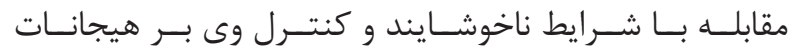

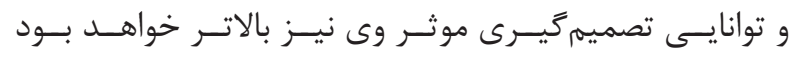

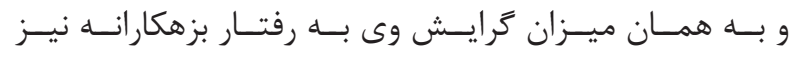

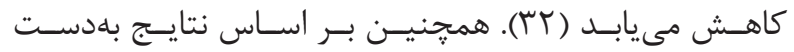

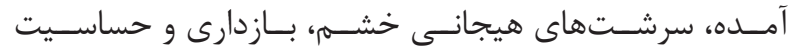

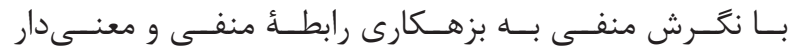

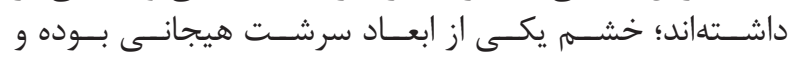

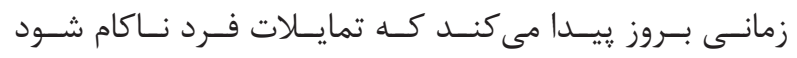

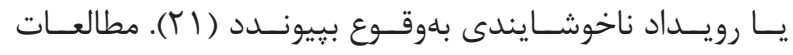

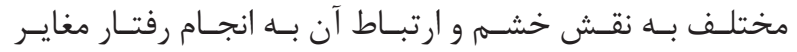

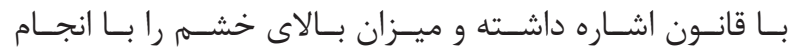

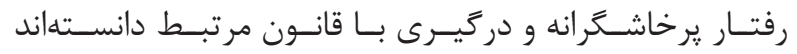

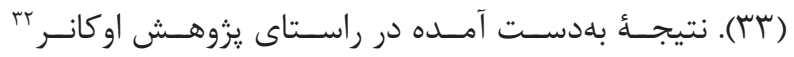

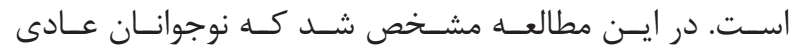

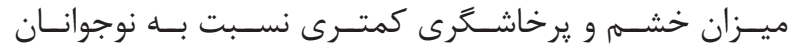

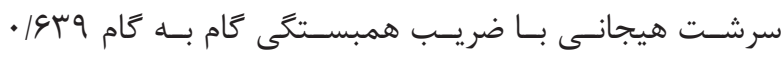

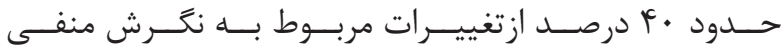

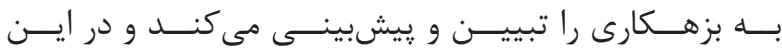

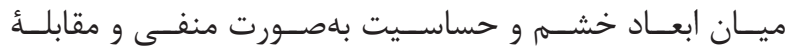

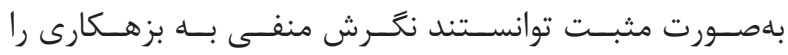

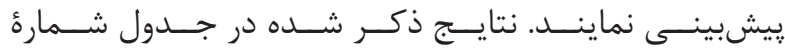

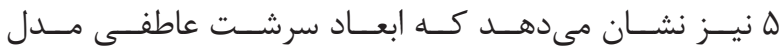

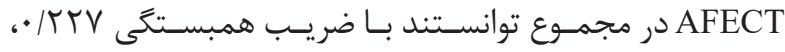

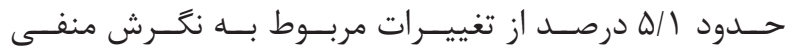

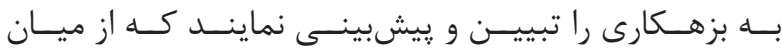

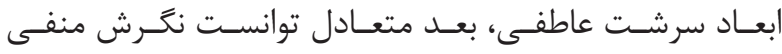

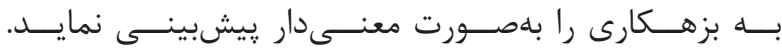

\section{بحث و نتيجهة تيرى}

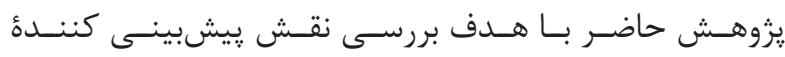

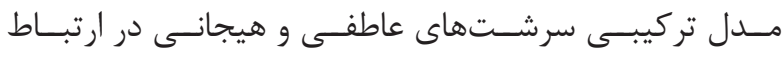

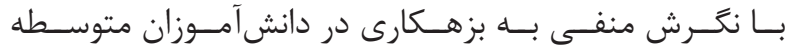

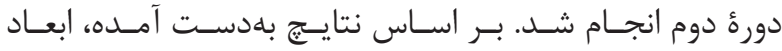

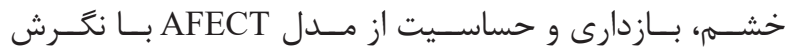

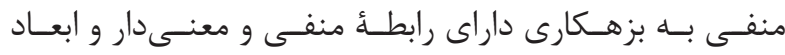

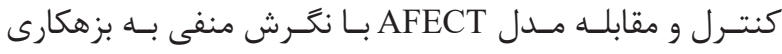

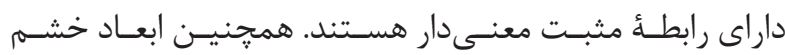

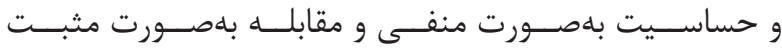

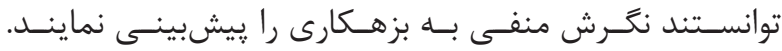

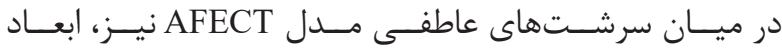

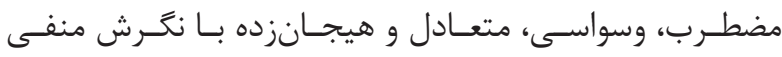

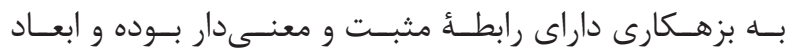

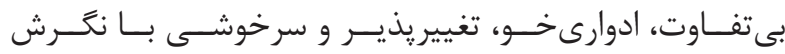

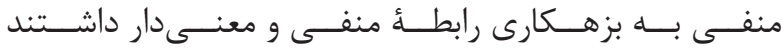

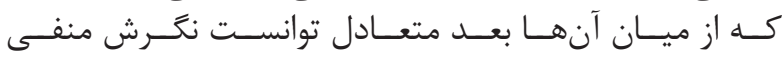

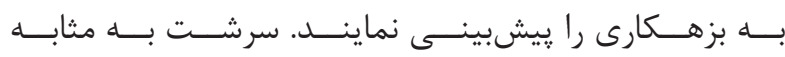

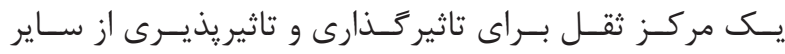

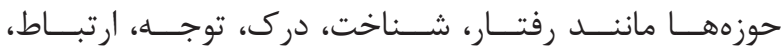

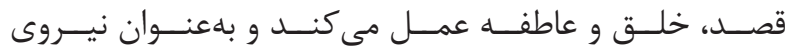

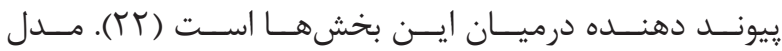

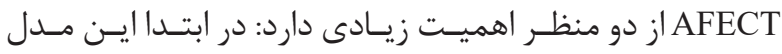

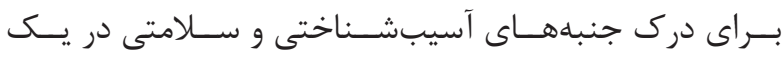

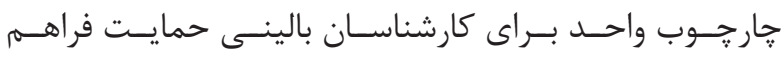

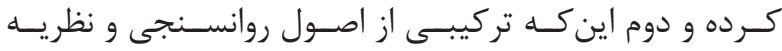

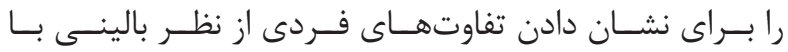

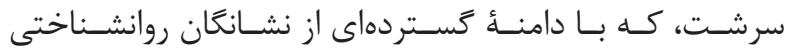

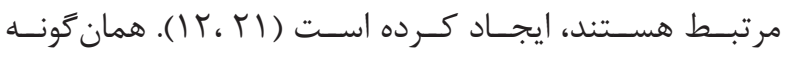

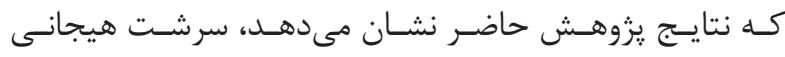

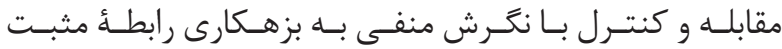

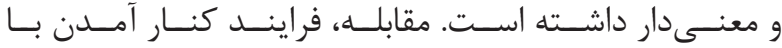

${ }^{31}$ Ruchkin, Eisemann \& Hagglof

${ }^{32} \mathrm{O}$ 'Connor 


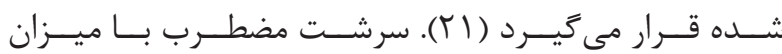

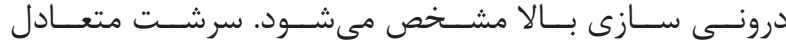

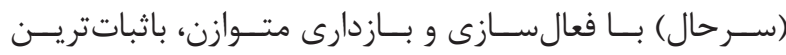

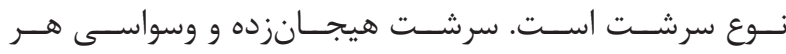

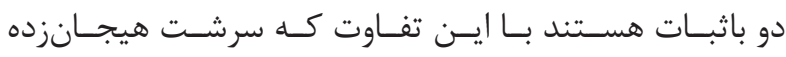

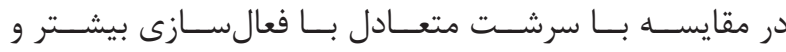

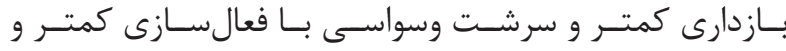

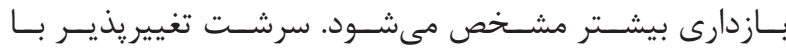

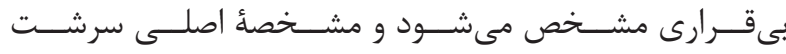

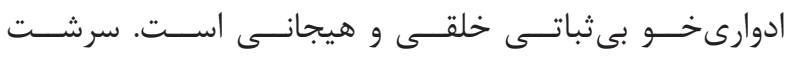

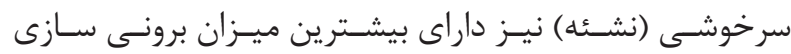

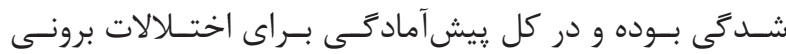

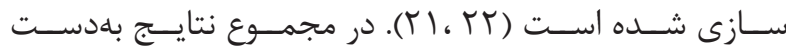

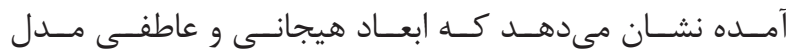

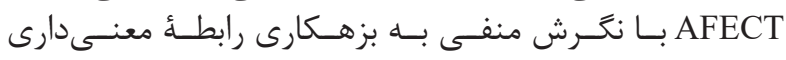

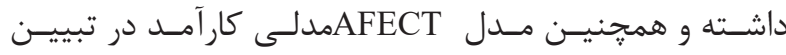

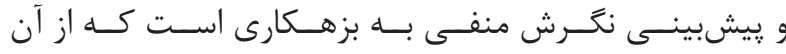

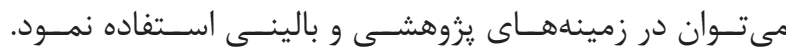

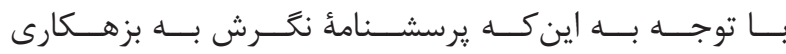

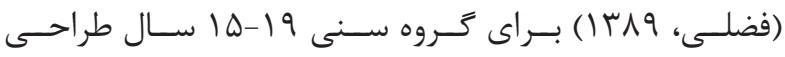

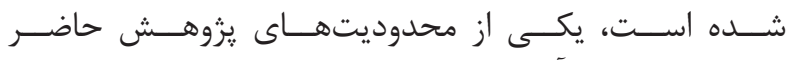

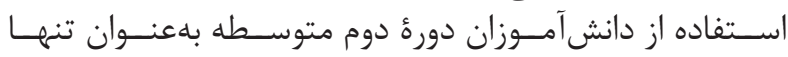

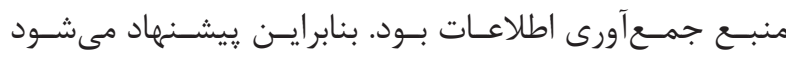

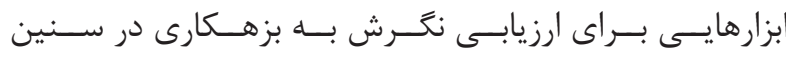

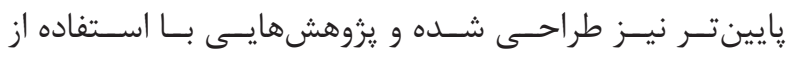

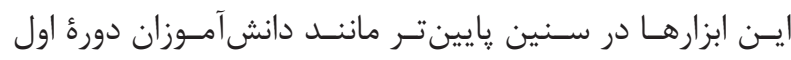

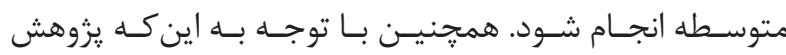

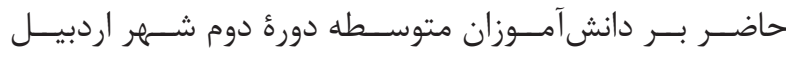

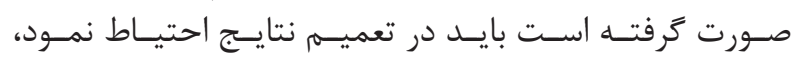

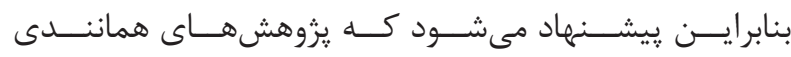

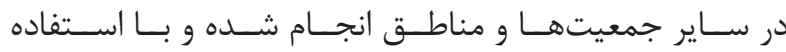

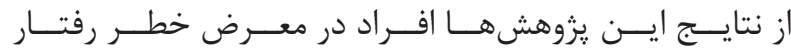

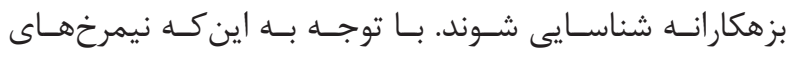

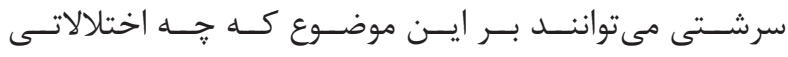

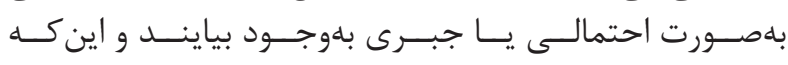

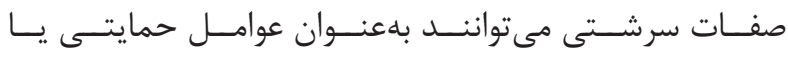

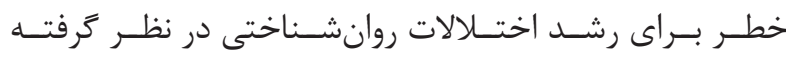

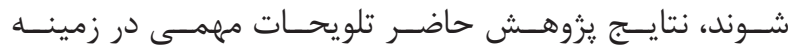

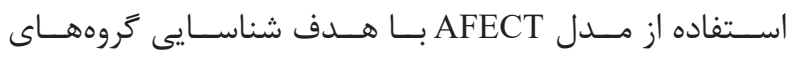

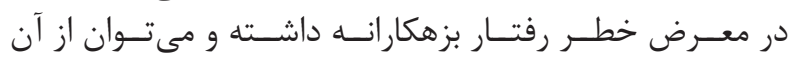

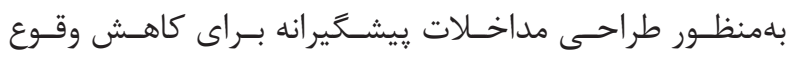

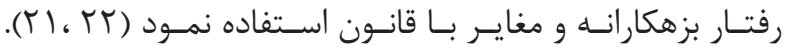

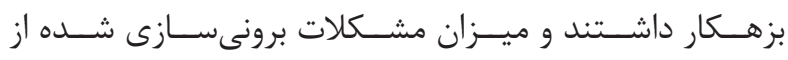

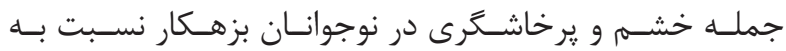

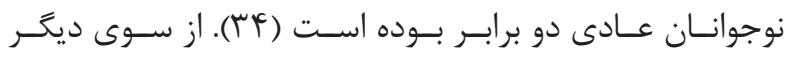

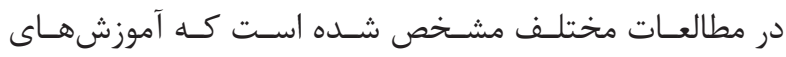

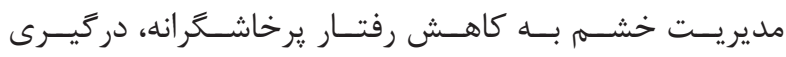

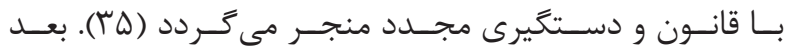

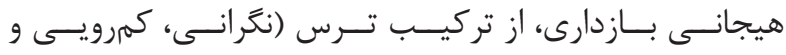

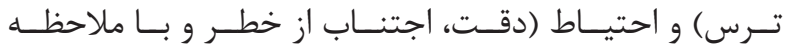

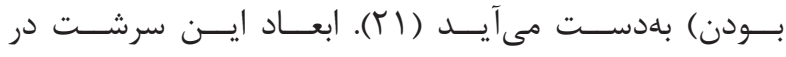

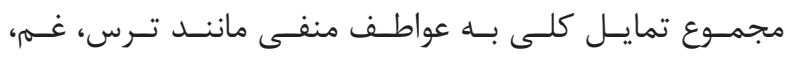

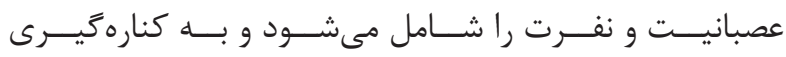

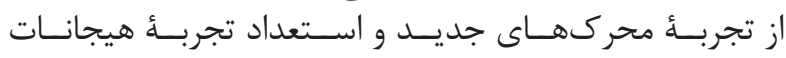

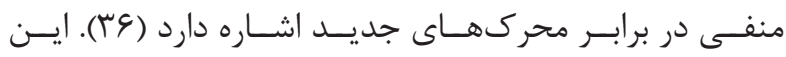

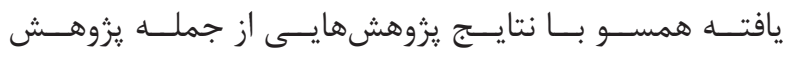

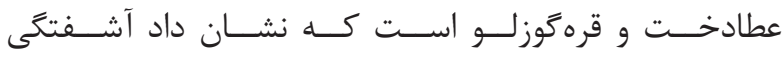

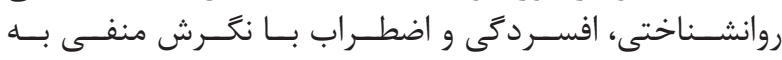

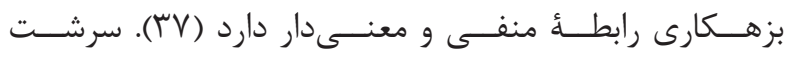

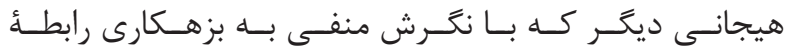

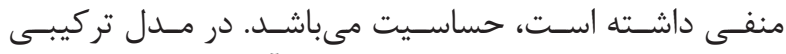
AFECT

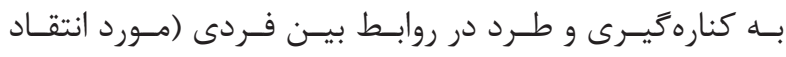

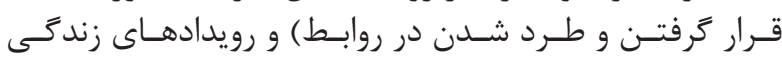

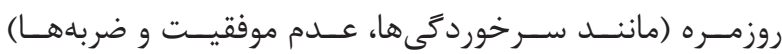

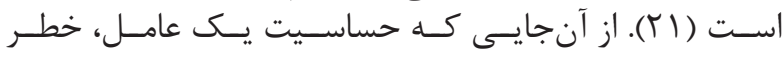

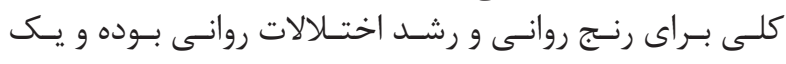

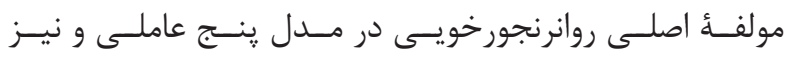

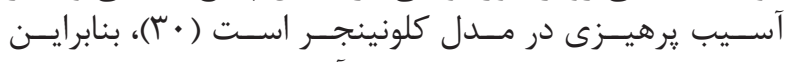

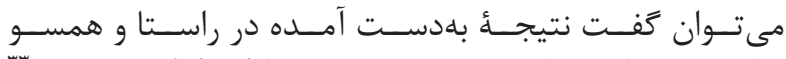

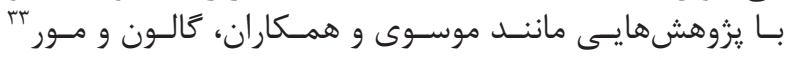

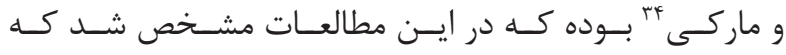

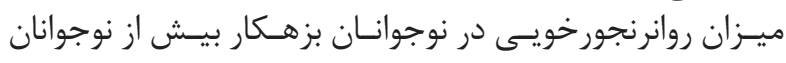

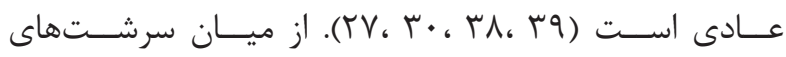

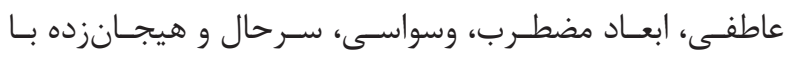

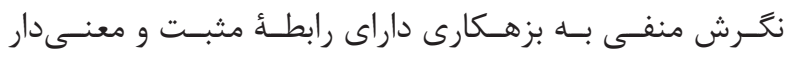

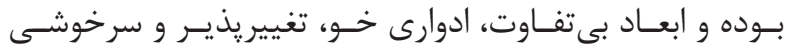

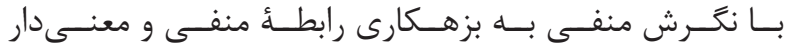

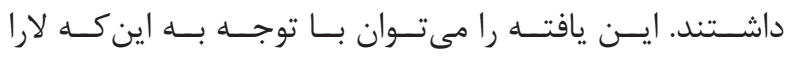

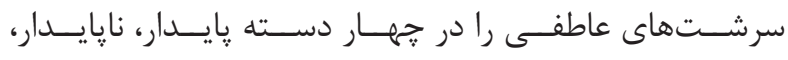

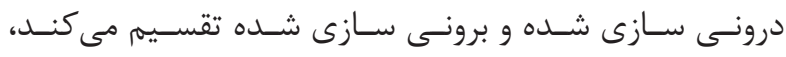

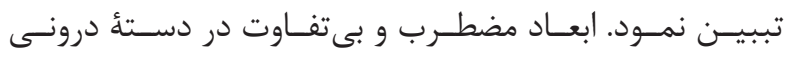

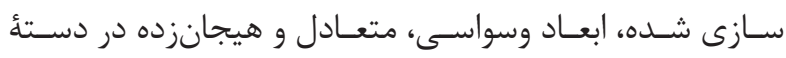

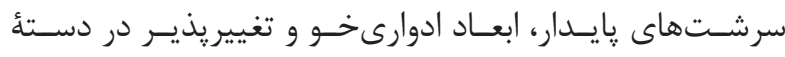

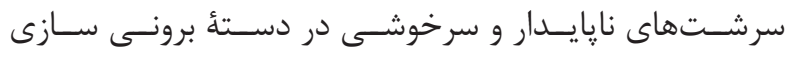


1. Vermeiren R. Psychology pathology and delinquency in adolescent: a descriptive and developmental perspective. Clin psychology review. 2003; 23:278-318.

2. Urbaniok F, Endrass J, Astrid R, Moll T. Violent and Sexual Offences: A Validation of the Predictive Quality of the PCL: SV in Switzerland. International Journal of Law and Psychiatry. 2006; 14: 45-57.

3. American Psychiatric Association (APA). Diagnostic and Statistical Manual of Mental Disorders (5th ed.) Washington, DC: American Psychiatric Association. 2013; P. 210-15.

4. He N, Marshall IH. A Multi-City Assessment of Juvenile Delinquency in the U.S.: A Continuation and Expansion of the International Self-Report Delinquency Study (ISRD), 2012; P.47-50.

5. Rajabipour M. Student delinquency and the possibility of social prevention of growth-oriented, Social Knowledge Quarterly. 2009; 12: 128-58.

6. Cohen MA, Piquero AR. New evidence on the monetary value of saving a high risk youth. Journal of Quantitative Criminology. 2009; 25: 25-49.

7. Lopes GD, Krohn AJ, Lizotte NM, Schmidt BV. Labeling and cumulative disadvantage the impact of formal police intervention on life chances and crime during emerging adulthood. Journal of Crime \& Delinquency. 2012; 58(3): 456-88.

8. Karimian H. A look at juvenile delinquency. Journal of Crime Prevention Studies. 2008; 17: 141-56.

9. Karbalaei F, Afrooz G, Raghibi M. Investigation of biological, personality and IQ characteristics in juvenile delinquents in Sistan and Baluchestan province. Research in Educational Systems. 2016; 33: 189-208.

10. Rettew DC, McKee L. Temperament and its role in developmental psychopathology. Harv Rev Psychiatry. 2005; 13(1):14-27.

11. Walters GD. Pathways to early delinquency: Exploring the individual and collective contributions of difficult temperament, low maternal involvement, and externalizing behavior. Journal of Criminal Justice. 2015; 42(4): 321-26.

12. Lara DR, Bisol LW, Brunstein M G, Reppold CT, de Carvalho H W, Ottoni GL. The Affective and Emotional Composite Temperament (AFECT) model and scale: A system-based integrative approach, Journal of Affective Disorders. 2012; 140: 14-37.
منابع

13. Shafiee M; Basharpour S, Heydari Rad H. The role of emotional-emotional nature in predicting symptoms of post-traumatic stress disorder in people with disabilities due to landmine explosions. Journal of Veterinary Medicine. 2017; (3) 9: 25-39.

14. Burt SA, Donnellan MB. Personality correlates of aggressive and non-aggressive antisocial behavior. Personality and Individual Differences. 2008; 44: 53-63.

15. Corf YL, Toupin J . Comparing persistent juvenile delinquents and normative peers with the five-factor model of personality. Journal of Research in Personality. 2009; 43: 1105-08.

16. Chelbianlu Gh, Abdi R, Sheikh S. The role of the combined emotional and emotional nature model (AFECT) in predicting the symptoms of pervasive anxiety disorder, Journal of psychological methods and models. 2018; (27) 8: 65-81.

17. Amodio DM, Master SL, Yee CM, Taylor. Neurocognitive components of the behavioral inhibition and activation systems: Implications for theories of self-regulation. Psychophysiology. 2008; 45: 11- 19.

18. MacDonald C, Bore M, Munr. D. Values in action scale and the Big 5: An empirical indication of structure. Journal of Research in Personality. 2008; 42: 787- 99.

19. Cloninger CR. Temperament and Personality. Current Opinion in Neurobiology. 1994; 4: 226- 73.

20. Clark LA, Watson D. Tripartite model of anxiety and depression: evidence and taxonomic implications. Journal of Abnormal Psychology. 1991; 100: 316-36.

21. Lara RD, Ottoni LG, Brunstein GM, Frozi J, Carvalho WH, Bisol WL. Development and validity of the Brazilian internet study on temperament and psychopathology (BRAINSTEP), Journal of Affective Disorders. 2012; 141(2-3): 390-98.

22. Basharpour S. personality psychology. Mohaghegh Arbeili University Press, Ardabil, 2017; P. 68-99.

23. Fazli V. Investigating the Relationship between Parents' Educational Methods and Attitudes towards Delinquent Behaviors among Adolescents aged 15 to 18 in Tehran, Quarterly Journal of Crime Prevention Studies. 2010; (14)5: 79-112.

24. Evans SZ, Simons LG, Simons AM. Factors that Influence Trajectories of Delinquency Throughout Adolescence. Empirical Research. 2015; 45: 156-71.

25. Farrington DP, Ttofi GM. Systematic reviews of explanatory risk factors for violence, 
offending, and delinquency. Aggression and Violent Behavior. 2017; 33: 24-36.

26. DeLisi M, Bryanna MD, Fully. HF, Vaughn MG. The effects of temperament, psychopathy, and childhood trauma among delinquent youth: A test of DeLisi and Vaughn's temperamentbased theory of crime, International Journal of Law and Psychiatry. 2018; 57: 53-63.

27. Mousavi V, Rezaei S, Shiri, M, Abd Khodaei M. The effect of delinquency and sex on parenting styles, attachment styles and personality traits of adolescents. Journal of Psychology. 2015; (2)78: 206-22.

28. Sadri S, Pezeshk S, Tayeb Lee, M. Characteristic characteristics and defensemechanisms ofmale offenders of the Correctional Center based on the type of crime. Journal of Exceptional People. 2016; (6)23: 179-97.

29. Ahmadi AD, Pour Naghash Tehrani S, Saberi SM, Arjmand $\mathrm{Nia} \mathrm{A}$. Investigation of personality traits, family status, mental health and IQ of delinquent and socially disadvantaged adolescents in Tehran, Journal of Psychological Sciences. 2018; 66: 228-45.

30. Chelbianlu Gh, Abdi R, Rasoulzadeh M, Sheikh, S. Investigation of Psychometric Characteristics of Combined Scale of Emotional and Emotional Nature (AFECT) in Students. New Psychological Research. 2016, 11(42): 51-71.

31. Ruchkin V, Eisemann M, Hagglof B. Coping Styles Delinquent of Adolescents and Controls: The Rol of Personality and Parental Rearing. Journal of youth and adolescent. 2005; 28(6): 705-17.
32. Fuscaldo VL, Bisol WL, Lara RD. How emotional traits and affective temperaments relate to cocaine experimentation, abuse and dependence in a large sample. Addictive Behaviors. 2013, 38: 1859-64.

33. Karimi H, Hemmati Sabet A, Haghighi M, Ahmad Panah M. Comparison of the effectiveness of group training on anger management and communication skills on the level of aggression of addicts to cannabis in Hamadan city prison, Behavioral Sciences Research. 2014; 11(2): 129-38.

34. O’Connor FW, Lovell D, Brown L. Implementing residential treatment for prison inmates with mental illness. Arch Psychiatr Nurs. 2012; 16(5): 232-8.

35. Ireland JL. Anger management therapy with young male offenders: An evaluation of treatment outcome. Aggressive Behavior. 2014; 30(2): 174-85.

36. Hossein Khanzadeh AA, Taher M. Analytical study of personality traits of delinquent adolescents. Journal of Criminal Law. 2014; 5(1): 31-53.

37. Atadakht A, Qaraguzlu, N. The role of depression, anxiety and stress in male students in predicting their positive and negative attitudes towards delinquency, school psychology. 2018; 7(2): 115-130.

38. Gullone E, Moore S. Adolescent risktaking and the five-factor model of personality. Journal of Adolescence. 2000; 23: 393-407.

39. Markey CN, Markey PM, Tinsley BJ. Personality, puberty, and preadolescentgirlsriskybehaviors: examining the predictive value if the five-factor model of personality. Journal of Research in Personality. 2003; 37: 405-19. 